\title{
Composition fluctuations in a non-critical binary polymer blend studied by ultrasonic and light scattering experiments
}

\author{
Susanne Eckert, ${ }^{a}$ Sven Hoffmann, ${ }^{a}$ Gerhard Meier ${ }^{b}$ and Ingo Alig*a \\ a Deutsches Kunststoff-Institut, D-64289 Darmstadt, Schloßgartenstr. 6, Germany \\ b IFF “Weiche Materie”, FZ-Jülich, Postfach 1913, D-52425 Jülich, Germany
}

Received 6th December 2001, Accepted 29th January 2002
First published as an Advance Article on the web 15th May 2002

A non-critical mixture of polyethylene glycol (PEG600, $M=600 \mathrm{~g} \mathrm{~mol}^{-1}$ ) and polypropylene glycol (PPG1000, $M=1000 \mathrm{~g} \mathrm{~mol}^{-1}$ ) was investigated by ultrasonic and light scattering experiments in the one-phase region. The mass fraction of polypropylene glycol in the non-critical mixture was $y_{\mathrm{PPG}}=0.365$. The explored temperature and frequency range of the ultrasonic experiment was $0.1 \leq T-T_{\mathrm{P}} \leq 17.3 \mathrm{~K}$ and $0.4 \mathrm{MHz} \leq f \leq 30 \mathrm{MHz}\left(T_{\mathrm{P}}\right.$ : phase separation temperature). The frequency dependence of the ultrasonic attenuation of the non-critical mixture shows a relaxation behaviour typical for composition fluctuations. The data can be analysed by the dynamic scaling theory of Bhattacharjee and Ferrell which was formally extended to the non-critical case using the concept of a pseudospinodal temperature. The characteristic time scale of the concentration fluctuations is described by a frequency $\omega_{D}=2 D / \xi^{2}$ where $D$ is the mutual diffusion coefficient and $\xi$ is the correlation length. In the frame of the pseudospinodal concept the temperature dependence of the characteristic frequency is expressed by $\omega_{D}=\omega_{0} \varepsilon^{z \nu}$ with $\varepsilon=\left(T-T_{\mathrm{PS}}\right) / T_{\mathrm{PS}}\left(T_{\mathrm{PS}}\right.$ : pseudospinodal temperature, $\varepsilon$ : reduced temperature, $\omega_{0}$ : critical amplitude, $z \nu$ : critical exponent). The temperature dependence of the frequency $\omega_{D}$ was determined by the ultrasonic spectra. From this data, using mean field exponents, a value of $\omega_{0}=9.4 \mathrm{MHz}$ was estimated which is comparable to that of the critical mixture. The description of the ultrasonic data with mean field exponents is justified by the results of dynamic light scattering.

\section{Introduction}

Ultrasonic experiments are an important tool for investigating relaxation processes in condensed matter. ${ }^{1}$ The ultrasonic behaviour of critical fluids has been studied extensively in the last four decades, both experimentally and theoretically. ${ }^{1-15}$ The experimental data are explained by the coupling of the sound wave with critical order parameter fluctuations. The ultrasound attenuation in binary mixtures can be described by the following mechanism: Because of alternate adiabatic compression and expansion of the fluid the local temperature and the pressure dependent critical temperature will change. Due to the lagged response of the concentration fluctuations energy is dissipated.

For a mixture of critical composition, the time scale of the decay of concentration fluctuations is characterized by a frequency $\omega_{D}=2 D / \xi^{2}$ where $\xi$ is the correlation length of critical concentration fluctuations and $D$ is the mutual diffusion coefficient. The temperature dependence of the frequency $\omega_{D}$ is described by a function $\omega_{D}=\omega_{0} \varepsilon^{z \nu}$, with $\varepsilon=\left(T-T_{\mathrm{C}}\right) / T_{\mathrm{C}}$ being the reduced temperature, $\omega_{0}$ the critical amplitude, and $z \nu$ the critical exponent.

There are several ultrasonic investigations on low molecular weight mixtures of critical composition ${ }^{2-5}$ and some investigations of polymer solutions. ${ }^{6,7}$ We previously reported on ultrasonic experiments on a critical polymer mixture. ${ }^{16}$ It could be shown that the ultrasonic measurements of a critical mixture of polyethylene glycol $\left(M=400 \mathrm{~g} \mathrm{~mol}^{-1}\right)$ and polypropylene glycol $\left(M=1000 \mathrm{~g} \mathrm{~mol}^{-1}\right)$ can be interpreted by the dynamic scaling theory for low molecular weight binary critical mixtures of Bhattacharjee and Ferrell. ${ }^{11}$ Although there are some light scattering ${ }^{17-21}$ and ultrasonic ${ }^{22}$ measurements of non-critical low molecular weight mixtures, to our knowledge no measurements of the composition fluctuations of non-critical polymer mixtures by ultrasonic attenuation or light scattering have been reported so far.

There are two concepts for the description of the temperature dependence of the correlation length of non-critical mixtures in the vicinity of the miscibility gap: the pseudospinodal concept, used for binary fluid systems first by $\mathrm{Chu}$ et al., ${ }^{21}$ and the model of parametric equations of state. ${ }^{23,24}$ The second model gives an expression for the correlation length of composition fluctuations apart from the critical point but does not make a statement on the diffusion coefficient. Thus, the pseudospinodal concept remains as a description of the temperature-dependent diffusion coefficient of non-critical mixtures. For the description of the ultrasonic behaviour, an extension of the Bhattacharjee-Ferrell theory for the ultrasonic attenuation of mixtures along the coexistence curve was proposed by Ferrell. ${ }^{25}$

The aim of this paper is to test the possibility to formally extend the Bhattacharjee-Ferrell theory to non-critical (polymer) mixtures by scaling the ultrasonic data to a pseudospinodal temperature instead of the critical temperature. Therefore it is necessary to study the applicability of the pseudospinodal concept to polymer blends of non-critical composition.

In this paper, we report experimental studies of ultrasonic attenuation and light scattering in a non-critical binary polymer mixture of polyethylene glycol with a molecular weight of $M=600 \mathrm{~g} \mathrm{~mol}^{-1}$ (PEG600) and polypropylene glycol with a molecular weight of $M=1000 \mathrm{~g} \mathrm{~mol}^{-1}$ (PG1000). The mass concentration of PPG was $y_{\mathrm{PPG}}=0.365$. The paper is organized as follows: After a survey of the theory for light scattering and ultrasonic attenuation of critical mixtures (Sections II $\mathrm{A}$ and B), the extension to non-critical mixtures is described in Section II C. In Section III, a sketch of the experimental conditions is given. The phase diagram of the system is described in Section IV A. The light scattering data (Section IV B) enable 
the determination of the critical exponents which are used in the scaling functions of Bhattacharjee and Ferrell. The ultrasonic data (Section IV C) correspond to a fit of the scaling functions with the mean-field critical exponents. The paper is closed by some concluding remarks.

\section{Theory}

\section{A. Light scattering for critical mixtures}

1. Static properties. For a critical binary polymer mixture in the vicinity of the critical point of demixing, the scattered light intensity $I(\boldsymbol{q})$ mainly results from fluctuations of the concentration. This intensity $I(\boldsymbol{q})$ of the scattered light is proportional to the structure factor $S(\boldsymbol{q})$, the Fourier transform of the correlation function of the concentration $G(\boldsymbol{r})$ where $r=r_{1}-r_{2}$ is the distance between two points $r_{1}$ and $r_{2}$ in the scattering volume.

If the product of the absolute value of the scattering vector $q$ and the correlation length $\xi$ is not too large $(q \xi \ll 1)$, it is approximately correct to write an Ornstein-Zernike form for $G(\boldsymbol{r}):^{26}$

$$
G(\boldsymbol{r}) \sim \frac{\exp (-r / \xi)}{r} .
$$

In eqn. (1) the correlation length $\xi$ is introduced. By Fourier transform of eqn. (1), one gets the Ornstein-Zernike scattering law

$$
S(\boldsymbol{q})=S(\boldsymbol{q}=0)\left(1+q^{2} \xi^{2}\right)^{-1} .
$$

The temperature dependence of the correlation length $\xi$ is given by

$$
\xi=\xi_{0} \varepsilon^{-\nu},
$$

where $\nu$ is the critical exponent of the correlation length, and $\varepsilon=\left(T-T_{\mathrm{C}}\right) / T_{\mathrm{C}}$ is the reduced temperature $(\nu=0.63$ for the $3 \mathrm{~d}$-Ising universality class and 0.5 for the mean field model).

2 Dynamic properties. In a binary mixture, the central quasielastic component in the spectrum of scattered light is caused by the diffusive decay of concentration fluctuations. By measuring the time autocorrelation function of the scattered light intensity, the dynamic structure factor $S(\boldsymbol{q}, t)=\langle\delta \phi(\boldsymbol{q}, t) \delta \phi(-\boldsymbol{q}, 0)\rangle$ with $\delta \phi(\boldsymbol{q}, t)=\phi(\boldsymbol{q}, t)-\langle\phi\rangle$, the decay of the order parameter $\delta \phi$ can be obtained. For a single exponential decay of the dynamic structure factor, the decay rate $\Gamma(\boldsymbol{q})$ is defined by

$$
S(\boldsymbol{q}, t)=S(\boldsymbol{q}) \exp (-\Gamma(\boldsymbol{q}) t)=S(\boldsymbol{q}) \exp \left(-D(\boldsymbol{q}) q^{2} t\right),
$$

where $S(\boldsymbol{q})$ is the static structure factor and $D(\boldsymbol{q})$ is the collective diffusion coefficient.

As $T$ approaches the critical temperature $T_{\mathrm{C}}, S(\boldsymbol{q})$ diverges and $\Gamma$ goes to zero, known as critical slowing down. The measured decay rate contains, besides the critical part, a non-critical background. ${ }^{27,28}$

The value of the critical term $D_{\mathrm{C}}(\boldsymbol{q})$ from mode coupling theory is: ${ }^{29}$

$$
\begin{gathered}
D_{\mathrm{C}}(\boldsymbol{q})=\frac{\Gamma_{\mathrm{C}}(\boldsymbol{q})}{q^{2}}=R \frac{k_{\mathrm{B}} T}{6 \pi \eta_{\mathrm{S}} \xi} K(x), \\
K(x)=\frac{3}{4 x^{2}}\left[1+x^{2}+\left(x^{3}-x^{-1}\right) \arctan x\right],
\end{gathered}
$$

with $\eta_{\mathrm{S}}$ being the shear viscosity, $x=q \xi, K(x)$ the Kawasaki function, and $R$ is a universal dynamic amplitude ratio $\left(R=1.056\right.$ for $\left.T=T_{\mathrm{C}}{ }^{30}\right)$.
In the hydrodynamic limit $(q \xi \rightarrow 0)$ the diffusion coefficient $D_{\mathrm{C}}$ is given by

$$
\lim _{q \xi \rightarrow 0} D_{\mathrm{C}}=R \frac{k_{\mathrm{B}} T}{6 \pi \eta \xi}
$$

The temperature dependence of the diffusion coefficient in the hydrodynamic limit is given by

$$
D_{C}=D_{0} \varepsilon^{\nu^{*}}
$$

where $\nu^{*}$ is the critical exponent of the diffusion coefficient: $\nu^{*}=\nu\left(1+x_{\eta}\right)$, with $x_{\eta}=0$ for the mean field model; for the 3d-Ising model $x_{\eta}=0.054$ from a mode coupling approach ${ }^{31}$ and 0.065 from renormalization group theory. ${ }^{32}$

\section{B. Ultrasonic attenuation in critical mixtures}

Critical ultrasound attenuation in binary blends is founded in the lagged response of the critical concentration fluctuations to the pressure induced temperature variations $\mathrm{d} T_{\mathrm{C}} / \mathrm{d} P$ (pressure dependence of the critical temperature along the critical line) and $(\partial T / \partial P)_{S}$ (adiabatic temperature raise). Theoretical studies of critical ultrasound behaviour are based on calculations of the frequency dependent complex bulk viscosity $9,10,12$ or the frequency dependent complex specific heat. ${ }^{11,13,14}$

In this study, the measurements are compared with results of the dynamic scaling theory of Bhattacharjee and Ferrell, ${ }^{11}$ which was successful in describing experimental data of mixtures with low molecular weight components, especially in the high frequency limit. ${ }^{2,3}$ In this limit the dynamic renormalization group study of Kroll and Ruhland ${ }^{12}$ gives similar results. The model of Bhattacharjee and Ferrell has been applied successfully to a critical mixture of polyethylene glycol $\left(M=400 \mathrm{~g} \mathrm{~mol}^{-1}\right)$ and polypropylene glycol $(M=1000 \mathrm{~g}$ $\left.\mathrm{mol}^{-1}\right){ }^{16}$

A dynamic scaling argument for the complex heat capacity leads to a simple functional dependence of the attenuation $\alpha / f^{2}$ on frequency $f$ at the critical temperature $T_{\mathrm{C}}$ :

$$
\left(\alpha / f^{2}\right)_{T_{\mathrm{C}}}=A / u_{\mathrm{C}} f^{-(1+\tilde{\alpha} /(z \nu))}+B
$$

where $\tilde{\alpha}$ is the critical exponent of the specific heat of the critical mixture at constant pressure, $\nu$ is the critical exponent of the correlation length $\xi$, and $z=3+x_{\eta} . B$ is a phenomenologically added background term. In our case $B$ is identical to $(\alpha /$ $\left.f^{2}\right)_{\mathrm{bg}, T_{\mathrm{C}}}$, the background attenuation at the critical point calculated from the mean contribution of the pure components. $A$ is a frequency independent constant and $u_{\mathrm{C}}$ is the ultrasound velocity at temperature $T_{\mathrm{C}}$.

To describe the critical ultrasound behaviour in the whole frequency and temperature range, Bhattacharjee and Ferrell give a parameter-free relation for the reduced quantity $\left(\alpha_{\lambda}\right)$ $\left.\alpha_{\lambda, T_{\mathrm{C}}}\right)\left(\alpha_{\lambda}\right.$ : ultrasound attenuation per wavelength at a given temperature, $\alpha_{\lambda, T_{\mathrm{C}}}$ : its value at the critical temperature):

$$
\alpha_{\lambda} / \alpha_{\lambda, T_{\mathrm{C}}}=G(\Omega)
$$

with $\Omega=\left(\omega / \omega_{D}\right)$ ( $\Omega$ : reduced frequency, $\omega=2 \pi f, \omega_{D}$ : frequency of the concentration fluctuations). The authors give an approximated relation for the scaling function $G(\Omega)$ :

$$
G(\Omega)=\left[1+0.414\left(\Omega_{1 / 2} / \Omega\right)^{1 / 2}\right]^{-2}
$$

with $G\left(\Omega_{1 / 2}\right)=\frac{1}{2} ; \Omega_{1 / 2} \simeq 2.1$. $^{11}$

This relation can be compared directly with the experimental results. The only parameter which has to be known is the amplitude $\omega_{0}$ of the characteristic frequency, which is available from light scattering experiments or can be determined by fitting the ultrasonic data.

The frequency $\omega_{D}$ of the concentration fluctuations at temperatures away from the critical temperature can be obtained from eqns. (8)-(10): 


$$
\left(\alpha / f^{2}\right)_{T}=A / u_{T} f^{-(1+\tilde{\alpha} /(z \nu))}\left(1+0.2393 \sqrt{\omega_{D} / f}\right)^{-2}+B_{T}
$$

where $u_{T}$ is the ultrasound velocity at temperature $T$ and $B_{T}$ is the background attenuation at temperature $T$. The temperature dependence of the frequency $\omega_{D}$ of the concentration fluctuations is described by a power law $\omega_{D}=\omega_{0} \varepsilon^{z \nu}$.

\section{Extension to non-critical mixtures}

The pseudospinodal concept was first used by Benedek ${ }^{33}$ for the description of the data at non-critical isochores of $\mathrm{SF}_{6}$ and was extended to non-critical binary mixtures by Chu et $a l^{21}$ The basic assumption of the pseudospinodal concept is that the temperature dependence of the thermodynamic and transport properties of a system at non-critical isochores can be described with similar power laws as at the critical isochore. It is assumed that at non-critical isochores these properties diverge at a pseudospinodal temperature $T_{\mathrm{PS}}$. The pseudospinodal temperature $T_{\mathrm{PS}}$ is an empirical parameter and can be determined by a fit of a power law to the data. The correlation length of a non-critical mixture, for example, is assumed to obey to the following equation:

$$
\xi=\tilde{\xi}_{0}\left(\frac{T-T_{\mathrm{PS}}}{T_{\mathrm{PS}}}\right)^{-\tilde{\nu}},
$$

The gas-liquid transition of one-component systems ${ }^{33}$ and the phase behaviour of low molecular weight binary mixtures $^{17-21,34,35}$ can be described satisfactorily in the frame of the empirical pseudospinodal concept.

By combining the Bhattacharjee-Ferrell theory and the pseudospinodal concept, we assume that the ultrasonic attenuation of a non-critical mixture $\alpha_{\lambda}{ }^{\text {n.c. }}$ can be related to its attenuation at the pseudospinodal temperature $T_{\mathrm{PS}}$ :

$$
\begin{gathered}
\alpha_{\lambda}^{\text {n.c. }} / \alpha_{\lambda, T_{\mathrm{PS}}}^{\text {n.c. }}=G\left(\Omega_{\text {n.c. }}\right), \\
\Omega_{\text {n.c. }}=2 \pi f / \omega_{D}^{\text {n.c }}, \omega_{D}^{\text {n.c. }}=\omega_{0}^{\text {n.c. }} \varepsilon^{z \nu}, \varepsilon=\left(T-T_{\mathrm{PS}}\right) / T_{\mathrm{PS}},
\end{gathered}
$$

where $\alpha_{\lambda}{ }^{\text {n.c. }}=$ ultrasound attenuation of the non-critical mixture; $\alpha_{\lambda, T_{\mathrm{PS}}}{ }^{\text {n.c. }}=$ ultrasound attenuation of the non-critical mixture at $T_{\mathrm{PS}}$; the superscript or subscript, respectively, n.c. corresponds to the non-critical mixture. In contrast to eqn. (9) where the reduced frequency $\Omega$ is related to the critical temperature, the reduced frequency $\Omega_{\text {h.c. }}$ of the non-critical mixture is related to the pseudospinodal temperature $(\varepsilon=$ $\left.\left(T-T_{\mathrm{PS}}\right) / T_{\mathrm{PS}}\right)$.

In this framework the frequency dependence of $\alpha / f^{2}$ at the pseudospinodal temperature is assumed to be:

$$
\left(\alpha / f^{2}\right)_{T_{\mathrm{PS}}}^{\text {n.c. }}=A^{\text {n.c. }} / u_{\text {n.c }} f^{-(1+\tilde{\alpha} /(z \nu))}+B,
$$

with the same meanings of the parameters as in eqn. (8). In the same way we formally extend eqn. (11) to the non-critical case:

$$
\begin{aligned}
\left(\alpha / f^{2}\right)_{T}^{\text {n.c. }}= & A^{\text {n.c. }} / u_{T}^{\text {n.c. }} f^{-(1+\tilde{\alpha} /(z \nu))} \\
& \times\left(1+0.2393 \sqrt{\omega_{\mathrm{D}}^{\text {n.c. }} / f}\right)^{-2}+B_{T}
\end{aligned}
$$

with the same meanings of the parameters as in eqn. (11) where the superscript n.c. indicates the non-critical mixture.

An alternative concept of data reduction for the scaling of non-critical ultrasonic data is the application of parametric equations of state introduced by Schofield ${ }^{23}$ and Josephson. ${ }^{24}$ The parameters $\varepsilon=\left(T-T_{\mathrm{C}}\right) / T_{\mathrm{C}}$ and $\phi-\phi_{\mathrm{C}}\left(\phi_{\mathrm{C}}\right.$ : critical composition) are related to parametric variables which depend on the phase diagram. The parameter transformation is determined by static scaling arguments, one of the transformations in accordance with the static scaling hypothesis is the restricted cubic model. ${ }^{36}$ For the parameter transformation, knowledge of the coexistence curve of the mixture is essential.
In our case, the coexistence curves of the critical and the non-critical mixture are different (see Section IV A). Due to this, an analysis of the light scattering and ultrasonic data in the frame of the model of parametric equations of state is not possible. For this analysis, the data of the critical reference mixture is needed. In this system, the reference state does not exist. So, the ultrasonic and light scattering data could only be analysed in the frame of the pseudospinodal concept.

\section{Experimental part}

\section{A. Samples}

The samples were commercial products obtained from Merck (Darmstadt) and Aldrich Chemie (Stuttgart). The molar mass distribution of the sample for light scattering experiments was characterized by MALDI mass spectroscopy (for polyethylene glycol (Merck): $M_{\mathrm{n}}=577 \mathrm{~g} \mathrm{~mol}^{-1}, M_{\mathrm{w}}=598 \mathrm{~g} \mathrm{~mol}, M_{\mathrm{w}} /$ $M_{\mathrm{n}}=1.036$, for polypropylene glycol (Aldrich): $M_{\mathrm{n}}=994 \mathrm{~g}$ $\left.\mathrm{mol}^{-1}, M_{\mathrm{w}}=1019 \mathrm{~g} \mathrm{~mol}^{-1}, M_{\mathrm{w}} / M_{\mathrm{n}}=1.025\right) .{ }^{37}$ The cloud point curve (see Fig. 1) was measured with a light scattering setup described previously. ${ }^{37}$ Additionally, the corresponding coexistence curves for the investigated non-critical mixture (mass concentration of PPG: $y_{\mathrm{PPG}}=0.365$ ) and for the critical mixture $\left(y_{\mathrm{PPG}}=0.46\right)$ were determined by differential refractometry. For the ultrasonic experiments the polypropylene glycol sample from Aldrich was replaced by a sample provided by Merck $\left(M_{\mathrm{n}}=970 \mathrm{~g} \mathrm{~mol} \mathrm{~mol}^{-1}, \quad M_{\mathrm{w}}=1000 \mathrm{~g} \mathrm{~mol} \mathrm{mo}^{-1}, M_{\mathrm{w}} /\right.$ $\left.M_{\mathrm{n}}=1.031\right)$.

\section{B. Light scattering experiments}

Proper amounts of the polymers were filtered through Millipore filters $(0.2 \mu \mathrm{m})$ into a dust free light scattering cell. The mixtures were prepared by weight. The homogenized sample was put into the light scattering setup and held at constant temperature until thermodynamic equilibrium was achieved. The light scattering experiments were performed with a commercial photometer (ALV, Langen). The light source was a $\mathrm{He}-\mathrm{Ne}$ laser (Spectra Physics, model 127) with a laser power of $35 \mathrm{~mW}$. The sample was measured in an angular range of $30^{\circ} \leq \theta \leq 150^{\circ}$, with $\theta$ being the scattering angle. With $q=(4 \pi n /$ $\lambda \sin (\theta / 2))$, this corresponds to a scattering vector range of $7.45 \times 10^{-3} \leq q \leq 2.78 \times 10^{-2} \mathrm{~nm}^{-1}$. The temperature was controlled electrically with an accuracy of $\pm 0.02 \mathrm{~K}$. The temperature range of the measurements was $0.05 \leq T-T_{\mathrm{P}} \leq 2.0 \mathrm{~K}$

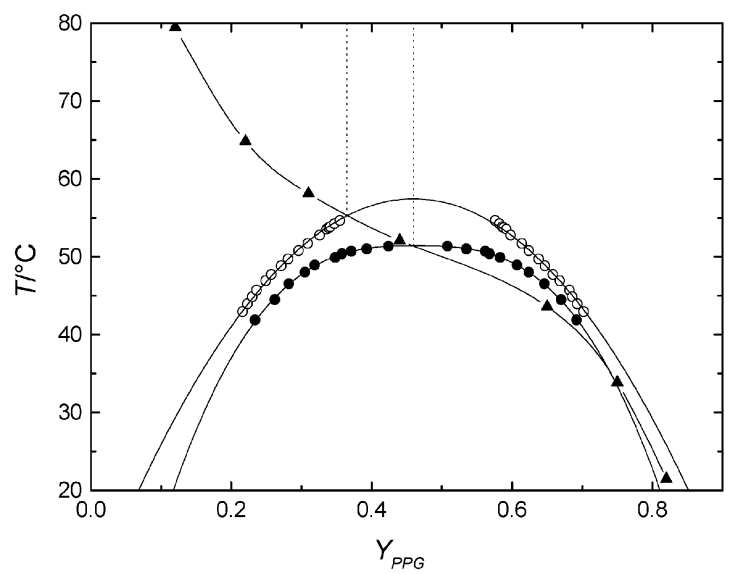

Fig. 1 Cloud point curve and phase coexistence curves of the mixture PEG600/PPG1000. Triangles: cloud point curve ${ }^{37}$ filled circles: phase coexistence curve of the mixture with $y_{\mathrm{PPG}}=0.46$ (critical mixture), open circles: phase coexistence curve of the mixture with $y_{\mathrm{PPG}}=0.365$. 0.365 . The dotted lines indicate the compositions of the mixtures in the one-phase region. 
( $T_{\mathrm{P}}$ : phase separation temperature). The incident and the scattered laser beam were polarized perpendicular to the scattering plane (VV geometry). The scattered intensity was measured by a photomultiplier. The intensity of the primary beam was measured by a photodiode. The dynamic light scattering experiments were performed with a correlator (ALV, model $5000 / \mathrm{E})$ in the same angular range as the static experiments.

In a quasielastic light scattering experiment, the desired autocorrelation function of the scattered field $g_{1}(\boldsymbol{q}, t)$ is related to the measured intensity time autocorrelation function $G_{2}(\boldsymbol{q}, t)$ through the Siegert relation

$$
g_{2}(\boldsymbol{q}, t) \equiv G_{2}(\boldsymbol{q}, t) /\langle I(\boldsymbol{q}, t)\rangle^{2}=1+f \alpha^{2}\left|g_{1}(\boldsymbol{q}, t)\right|^{2}
$$

where $\langle I(\boldsymbol{q}, t)\rangle$ is the mean intensity, $f$ is an instrumental factor, and $\alpha$ is the fraction of the totally scattered intensity arising from concentration fluctuations $\left(\alpha=I_{\text {c.f. }}(\boldsymbol{q}) / I(\boldsymbol{q})\right)$. In this case, the field correlation function $g_{1}(\boldsymbol{q}, t)$ is identified as the concentration autocorrelation function: $g_{1}(\boldsymbol{q}, t)=\langle\delta \phi(\boldsymbol{q}, t) \delta \phi(-\boldsymbol{q}, 0)\rangle /\left\langle|\delta \phi(\boldsymbol{q}, 0)|^{2}\right\rangle$, where $\left\langle|\delta \phi(\boldsymbol{q}, 0)|^{2}\right\rangle$ the mean square concentration fluctuations. It is further given by $^{38}$

$$
g_{1}(\boldsymbol{q}, t)=\frac{S(\boldsymbol{q}, t)}{S(\boldsymbol{q})}
$$

with the dynamic structure factor $S(\boldsymbol{q}, t)$ and the static structure factor $S(\boldsymbol{q})=\langle\delta \phi(\boldsymbol{q}, 0) \delta \phi(-\boldsymbol{q}, 0)\rangle$. The decay rate $\Gamma(\boldsymbol{q})$ of $S(\boldsymbol{q}, t)$ as defined in eqn. (4) is identical to the Rayleigh linewidth to be identified with the collective thermal decay rate of composition fluctuations $\delta \phi(\boldsymbol{q}, t)$. In the absence of mode coupling effects, the measured $\Gamma(\boldsymbol{q})=D q^{2}$ is related to the mutual diffusion coefficient $D$ as given by eqn. (4).

If the decay of the dynamic structure factor is not single exponential, the data can be analysed using an inverse Laplace transformation technique. There, $g_{1}(\boldsymbol{q}, t)$ is represented by

$$
g_{1}(\boldsymbol{q}, t)=\int_{0}^{\infty} \exp (-t / \tau) L(\tau) \mathrm{d} \tau,
$$

where $L(\tau)$ is the distribution of relaxation times. If $L(\tau)$ describes a single relaxation process, the relaxation time is given by the average

$$
\langle\tau\rangle=\int_{0}^{\infty} g_{1}(\boldsymbol{q}, t) \mathrm{d} t=\int_{0}^{\infty} \tau L(\tau) \mathrm{d} \tau,
$$

Then, $\langle\tau\rangle$ is related to the diffusion coefficient via $\langle\tau\rangle^{-1}=$ $\langle D\rangle q^{2}$. This procedure can be extended to distributions $L(\tau)$ describing separated relaxation processes.

\section{Ultrasound measurements}

The ultrasonic absorption was measured by a resonator method (frequency range: $0.4<f<1.5 \mathrm{MHz}$ ) and with the same resonator cell by a pulse transmission technique (frequency range: $6 \mathrm{MHz}<f<30 \mathrm{MHz}$ ) similar to the method described by Eggers and Funck. ${ }^{39}$ The temperature was controlled by an external thermostat with an accuracy of $\pm 0.02 \mathrm{~K}$. To avoid humidity, the ultrasonic cell was hold in a dry box filled with silica gel. This was necessary because of the hygroscopic character of the studied substances. The measurements were carried out in a temperature range of $0.1 \leq T-T_{\mathrm{P}} \leq 17.3 \mathrm{~K}$. Because of the wide temperature range, the cell alignment was checked carefully after each measurement.

\section{Results and discussion}

\section{A. Phase diagram}

In Fig. 1, the cloud point curve of the system PEG600/ PPG1000 is shown. The measurements are described in the literature. ${ }^{37}$ Together with the cloud point curve (triangles), the coexistence curves of the investigated non-critical mixture (mass fraction $y_{\mathrm{PPG}}=0.365$, open circles) and the critical mixture (mass fraction $y_{\mathrm{PPG}}=0.46$, solid circles), measured by differential refractometry, are plotted. The coexistence curves were measured to obtain the parameters necessary for a description with the model of parametric equations of state. For each differential refractometry measurement in the two phase region, approximately one day was allowed after the start of demixing and control measurements concerning the stability of the measurement data were made in order to exclude non-equilibrium effects.

In contrast to low molecular weight binary mixtures, cloud point curve and coexistence curves do not coincide and the mass fraction of the critical mixture is not equal to the composition of the mixture with the highest phase separation temperature. Also the coexistence curves of the two mixtures do not coincide. In general, this can be explained by a polydispersity of the compounds. ${ }^{40,41}$ In this case, the two-dimensional phase diagram of the system is just a quasi-binary section of the high dimensional phase space spanned by all components with different molar masses. Upon demixing, a fractionation of the polymers occurs for all mixtures except the critical mixture. ${ }^{41,42}$

On the other hand, the molar mass distribution of the samples is too narrow to explain the results (see Section III A). As the system has a relatively large amount of $\mathrm{OH}$ groups, the chains can build clusters by intermolecular hydrogen bonds. Hydrogen bonds were measured by infrared spectroscopy in PEG $^{43}$ and PPG. ${ }^{44,45}$ For PPG, ultrasound absorption ${ }^{46}$ and nuclear magnetic resonance measurements ${ }^{47}$ showed an additional low frequency relaxation process with characteristics typical for entanglement effects. The process occurred in samples with molar masses $M_{\mathrm{n}}>1300 \mathrm{~g} \mathrm{~mol}^{-1}$, far below the critical molar mass of PPG for entanglements $\left(M_{\mathrm{C}} \approx 7000 \mathrm{~g}\right.$ $\mathrm{mol}^{-1}$. The relaxation process disappeared by conversion of the hydroxy end groups into trimethylsilyl groups. The observed effect could be described by a model ${ }^{48}$ which assumed a formation of "supermolecules" by intermolecular end-toend hydrogen bonds with mean lifetimes long enough to form entanglements.

The formation of these clusters would lead to an "effective polydispersity" which can explain the difference between cloud point curve and coexistence curves and the difference between critical composition and maximum of the cloud point curve. The latter result was also found for the system PEG400/ PPG1000. ${ }^{16}$ As the PPG-molecules can also build clusters with PEG-molecules, the effective polydispersity could also be different for different mass concentrations $y_{\mathrm{PPG}}$ of the mixture. This aspect will be the subject of further investigations. ${ }^{49}$ It is interesting that the concentrations of the maxima of the coexistence curves are similar for the critical mixture (solid circles) and the non-critical mixture (open circles).

The phase diagram of the mixture used for the ultrasonic measurements is shifted to lower temperatures by about 20 $\mathrm{K}$. This is related to the fact that for the ultrasonic measurements, PPG samples from a different supplier were used (see Section III A). However, it is generally assumed that small differences in the chemical compositions may influence the phase diagram strongly whereas the critical fluctuations as a universal feature depend only on the spatial correlation function of the concentration fluctuations. Therefore the use of reduced variables enables us to compare the results from different samples.

\section{B. Light scattering}

1. Diffusion coefficient. The decay rate or Rayleigh linewidth $\Gamma$ of the concentration fluctuations was obtained by inverse Laplace transformation. The diffusion coefficient in the hydrodynamic limit is obtained from the measured Ray- 
leigh linewidth with eqns. (5) and (6) as ${ }^{50}$

$$
D \cong D_{\mathrm{C}}=\frac{\Gamma(\boldsymbol{q})}{q^{2}} \frac{1}{K(x)}
$$

In Fig. 2, a typical intensity correlation function $g_{2}(t)-1=$ $f \alpha^{2}\left|g_{1}(\boldsymbol{q}, t)\right|^{2}$ (see eqn. (16)) is shown for a scattering angle of $\theta=60^{\circ}$. The correlation function exhibits a two-step decay. In the inset of Fig. 2, the analysis of this correlation function with inverse Laplace transformation using the CONTIN algorithm fitting the square of the first order correlation function is represented. The obtained distribution function clearly shows two distinct maxima in the distribution of relaxation times $L(\tau)$.

The slower relaxation process is attributed to the diffusion of hydrogen bond clusters. ${ }^{49}$ This is supported by an analysis based on the Stokes-Einstein equation, ${ }^{49} D=k_{\mathrm{B}} T /\left(6 \pi \eta R_{\mathrm{g}}\right)$, where $R_{\mathrm{g}}$ is the radius of gyration) which gives an almost temperature independent value of $R_{\mathrm{g}}$ of about $400 \mathrm{~nm}$. The attribution to hydrogen bond clusters is also confirmed by an investigation of mixtures of PEG600/PPG1000 of different compositions in the one- and two-phase region which showed a dependence of the process on the composition of the mixture. ${ }^{49,51}$

The faster relaxation process is related to the composition fluctuations. This is justified by the power law dependence of the related diffusion coefficient (see Fig. 3). In Fig. 3, the diffusion coefficient is shown as a function of the reduced temperature $\varepsilon=\left(T-T_{\mathrm{PS}}\right) / T_{\mathrm{PS}}$. The fit of the exponential law $D=D_{0} \varepsilon^{\nu^{*}}$ was performed in two steps: first, $T_{\mathrm{PS}}$ was obtained by fitting a polynomial of second order to the data in a double logarithmic plot and minimizing the coefficient of second order. This yielded a value of $T_{\mathrm{PS}}=52.22^{\circ} \mathrm{C}$. Secondly, $\nu^{*}$ and $D_{0}$ were obtained by a two-parameter fit of the function $D=D_{0} \varepsilon^{\nu^{*}}$ with the value of $T_{\mathrm{PS}}$ fixed. The results of the fitting procedure are $\nu^{*}=0.50$ and $D_{0} 1.22 \times 10^{-7} \mathrm{~cm}^{2} \mathrm{~s}^{-1}$ (solid line). On the basis of these results, we conclude that the mixture shows mean field behaviour. Therefore, in the following we used mean field critical exponents for the scaling of the static light scattering and the ultrasonic data.

2. Correlation length. The results of the static light scattering measurements are shown in the upper part of Fig. 4. The reciprocal value of the scattered intensity is plotted as a function of the square of the scattered wave vector (Ornstein-Zer-



Fig. 2 Normalized intensity autocorrelation function $g_{2}(q, t)-1$ of the scattered light as a function of time. Two processes can be separated: The slower one is attributed to the diffusion of hydrogen bonded clusters, whereas the fast one is related to the composition fluctuations. In the inset, the results of an inverse Laplace transformation (CONTIN) is plotted, showing two well-separated maxima in the distribution function $L(\tau)$ of relaxation times $\tau$.

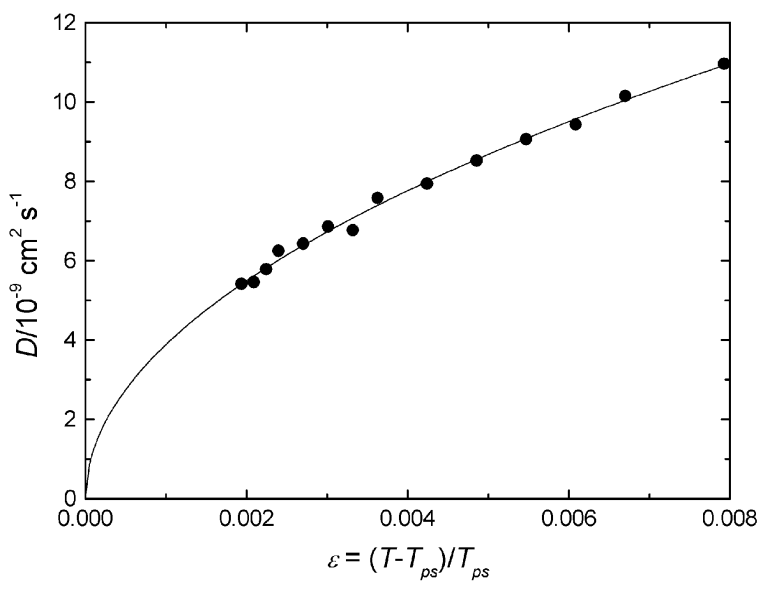

Fig. 3 Mutual diffusion coefficient $D$ as a function of the reduced temperature $\varepsilon$. The results of the fitting procedure (solid line) are: $T_{\mathrm{PS}}=52.22^{\circ} \mathrm{C}, \nu^{*}=0.50, D_{0}=1.22 \times 10^{-7} \mathrm{~cm}^{2} \mathrm{~s}^{-1}$.

nike (OZ) plot). The data can not be fitted by straight lines as expected for $\mathrm{OZ}$ behaviour of the $q$ range studied. This is caused by the occurrence of two relaxation processes measured by dynamic light scattering (see above) which are related to different length scales in the static experiment.

On the basis of the scattering intensities of the two processes extracted from the dynamic experiments we decomposed the two contributions of the total scattered intensity shown in Fig. 4, i.e. $I(\boldsymbol{q})=I_{\mathrm{fl} .}(\boldsymbol{q})+I_{\text {cluster }}(\boldsymbol{q})$ with $I_{\mathrm{fl} .}(\boldsymbol{q})$ and $I_{\text {cluster }}(\boldsymbol{q})$ being the contributions of the composition fluctuations and of the clusters, respectively. Our procedure was as follows:
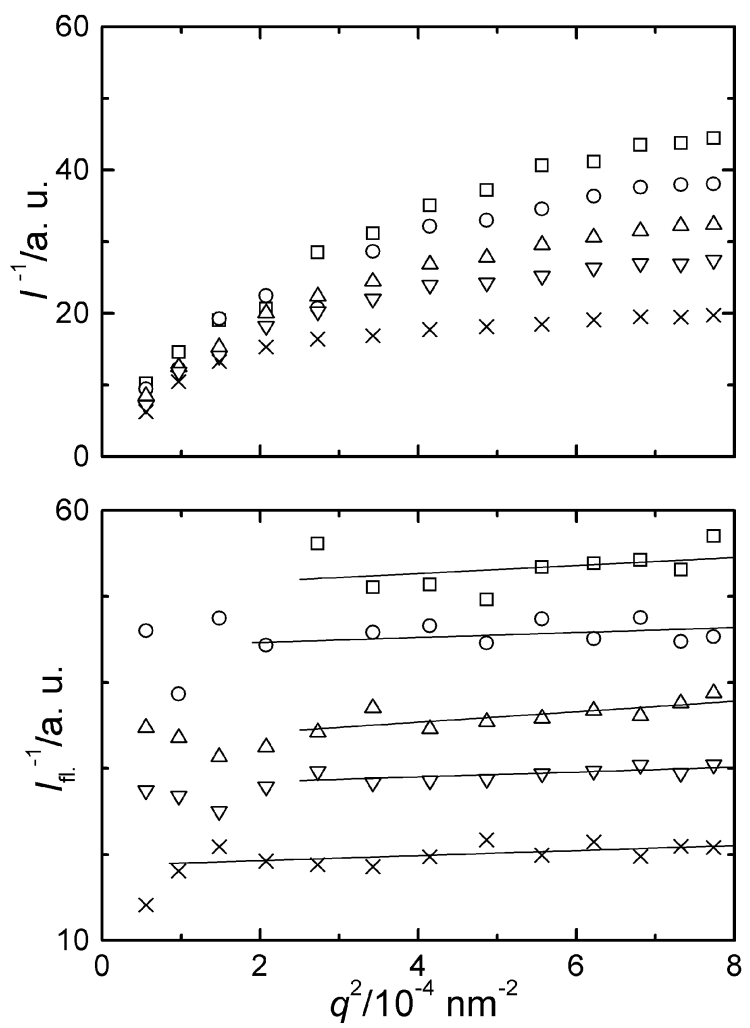

Fig. 4 Upper part: Ornstein-Zernike plot of the scattered light intensity for selected temperatures (squares: $T=54.8^{\circ} \mathrm{C}$, circles: $T=54.2^{\circ} \mathrm{C}$, up triangles: $T=53.6^{\circ} \mathrm{C}$, down triangles: $T=53.2^{\circ} \mathrm{C}$, crosses: $T=52.85^{\circ} \mathrm{C}$ ). Lower part: Ornstein-Zernike plot for the intensities $I_{\mathrm{fl}}$. due to the concentration fluctuations. In the lower part, the fits of the Ornstein-Zernike law yielding an estimate of the correlation length $\xi$ of the fluctuations (see Fig. 5) are also shown. 
The areas under the peaks shown, e.g., in the inset of Fig. 2 are proportional to the contributions of the related scattering processes. The fitting procedure of the correlation function yields these areas. From this information we can determine the proportion of every scattering process. Together with the information from static light scattering (done at the same time) we determined the absolute contribution (i.e. the scattering intensity) of every scattering process. The contribution of the scattered intensity from the composition fluctuations $I_{\mathrm{fl} .}(\boldsymbol{q})$ is shown in the lower part of Fig. 4 in an OZ plot. A fit of the Ornstein-Zernike law to the data yields the correlation length $\xi$ of the fluctuations. Due to the large error in the determination of the contribution of the slow process, the data show large scatter. Therefore a determination of the pseudospinodal temperature and the critical exponent for the correlation length from static light scattering data alone was not possible. The estimated correlation length is shown below in Fig. 5.

Since the correlation length determined by static light scattering is only a rough estimation, we alternatively calculated the correlation length from dynamic light scattering and measured viscosity data, using eqn. (6):

$$
\xi=R \frac{k_{\mathrm{B}} T}{6 \pi \eta D_{\mathrm{C}}} .
$$

The quality of the viscosity data for the calculation of the correlation length was checked by a comparison of the correlation length of the critical mixture of PEG600/PPG1000, determined by static light scattering on the one hand and by dynamic light scattering and viscosity data on the other hand. For this mixture, there exist reliable data from static light scattering. ${ }^{51}$ The correlation lengths of the critical mixture determined by these two methods are shown in the inset of Fig. 5 as a function of the reduced temperature. The data agree rather well. In Fig. 5, the correlation length determined by dynamic light scattering and viscosity data is shown for the non-critical mixture (solid triangles). As a comparison, the data from static light scattering are plotted (open triangles). These data show large scatter and cannot be used for the estimation of critical parameters. Thus, the function $\xi=\xi_{0} \varepsilon^{-\nu}$ $\left(\varepsilon=\left(T-T_{\mathrm{PS}}\right) / T_{\mathrm{PS}}\right)$ was fitted to the $\xi$ values calculated by eqn. (21) from the dynamic data. The fit with the fixed values $\nu=0.50$ and $T_{\mathrm{PS}}=52.22^{\circ} \mathrm{C}$ (values of $\nu$ and $T_{\mathrm{PS}}$ obtained by

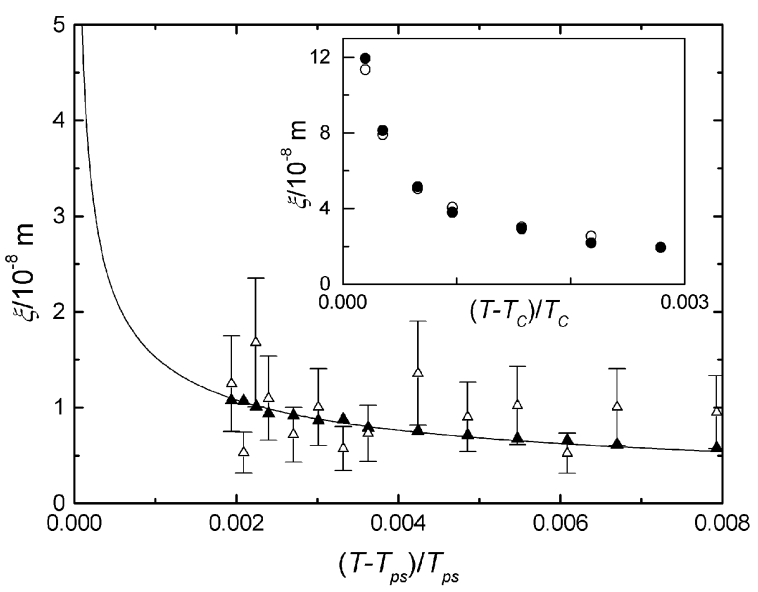

Fig. 5 Correlation length of the non-critical mixture determined by dynamic light scattering and viscosity data (filled triangles) as a function of the reduced temperature $\varepsilon=\left(T-T_{\mathrm{PS}}\right) / T_{\mathrm{PS}}$. The fitting procedure (solid line) with fixed values for $T_{\mathrm{PS}}$ and $\nu\left(T_{\mathrm{PS}}=52.22^{\circ} \mathrm{C}\right.$, $\nu=0.50)$ yields the amplitude of the correlation length $\xi_{0}$. For comparison, the results from static light scattering are shown (open triangles). Inset: Correlation length of the critical mixture determined by static light scattering (open circles) and dynamic light scattering and viscosity data (filled circles) as a function of the reduced temperature $\varepsilon=\left(T-T_{\mathrm{C}}\right) / T_{\mathrm{C}}$. the diffusion coefficient, see above) is also shown in Fig. 5. The fitting procedure yielded for the critical amplitude of the correlation length the result $\xi_{0}=0.48 \mathrm{~nm}$. From the results of the dynamic light scattering measurements, the amplitude of the relaxation frequency of the composition fluctuations was determined as $\omega_{0}=2 D_{0} / \xi_{0}^{2}=105 \mathrm{MHz}$.

\section{Ultrasonic attenuation}

Fig. 6 shows the measured attenuation $\alpha / f^{2}$ of the pure components (PEG600 and PPG1000) and the non-critical mixture $\left(y_{\mathrm{PPG}}=0.365\right)$ at two representative temperatures. The ultrasonic attenuation was measured at 18 different temperatures. In a classical fluid a longitudinal ultrasonic wave is attenuated by viscous damping and thermal conductivity. In this case, the frequency dependence of the ultrasonic attenuation coefficient can be described by the Kirchhoff-Stokes law as $\alpha \propto f^{2}$ (see, e.g., ref. 52). This behaviour is represented as a constant value in an $\alpha / f^{2}$ vs. $f$ plot. A decrease in $\alpha / f^{2}$ with increasing frequency can therefore be attributed to an additional relaxation process, since sound scattering can be almost excluded for our systems. The attenuation of the lower molecular weight PEG component deviates slightly from the behaviour of a classical liquid, the deviation is more pronounced for the PPG component.

The broad ultrasonic spectra obtained for PEG and PPG can be related to the Rouse-Zimm modes in polymeric systems, which yield the relation $\alpha / f^{2} \propto f^{n}$, with $n=-1 / 2$ for the Rouse theory ${ }^{53}$ and $n=-1 / 3$ for the Zimm theory ${ }^{54}$ which includes hydrodynamic interactions. For an estimation of the exponent $n$, a knowledge of the high frequency contribution $\left(\alpha / f^{2}\right)_{\infty}$ would be necessary. This contribution arises from relaxation modes related to molecular processes with a length scale smaller than the chain segments considered in the Rouse-


Fig. 6 Ultrasonic attenuation $\alpha / f^{2}$ as a function of frequency $f$ at the temperatures $50^{\circ} \mathrm{C}$ and $38^{\circ} \mathrm{C}$. Filled circles: experimental data from the non-critical mixture $\left(y_{\mathrm{PPG}}=0.365\right)$, open circles: experimental data from pure PEG600, open squares: experimental data from pure PPG1000. 
Zimm theory. However, due to the limited frequency range of our experiments a more detailed analysis of the ultrasonic spectra is difficult.

The mixture shows an excess attenuation with a significantly different behaviour as expected for Rouse-Zimm modes, depending only weakly on temperature. The features of the excess attenuation of the non-critical mixture are similar to those found for a critical mixture of PEG and PPG before, ${ }^{16}$ which could clearly be related to the contribution from critical composition fluctuations.

For a more detailed analysis, the fluctuation contribution of the non-critical mixture must be separated from the background contribution related to the pure components. The basis of this separation is the assumption that the excess attenuation of the mixture $\left(\alpha / f^{2}\right)_{\text {mix }}$ and the background contribution $(\alpha /$ $\left.f^{2}\right)_{\text {bg }}$ are additive:

$$
\alpha / f^{2}=\left(\alpha / f^{2}\right)_{\text {mix }}+\left(\alpha / f^{2}\right)_{\text {bg }} .
$$

Since there is no theoretically justified procedure to determine the background contribution, we tried two different possibilities, which are based on the data analysis of a critical mixture of PEG and PPG in: ${ }^{16}$

(i) Measurement of the attenuation of the pure components and subtraction of (a) their arithmetical mean contribution

$$
\left(\alpha / f^{2}\right)_{\mathrm{bg}}=\phi_{\mathrm{PPG}}\left(\alpha / f^{2}\right)_{\mathrm{PPG}}+\left(1-\phi_{\mathrm{PPG}}\right)\left(\alpha / f^{2}\right)_{\mathrm{PEG}}
$$

or (b) their reciprocal mean contribution

$$
\left(\alpha / f^{2}\right)_{\mathrm{bg}}^{-1}=\phi_{\mathrm{PPG}}\left(\alpha / f^{2}\right)_{\mathrm{PPG}}^{-1}+\left(1-\phi_{\mathrm{PPG}}\right)\left(\alpha / f^{2}\right)_{\mathrm{PEG}}^{-1},
$$

where $\phi_{\mathrm{PPG}}$ is the volume fraction of PPG in the mixture. The reciprocal construction is motivated by shear viscosity data in a polymeric mixture. ${ }^{50}$ The ultrasonic absorption can be related to a combination of the dynamic bulk viscosity $\eta_{\mathrm{V}}$ and shear viscosity $\eta_{\mathrm{S}}\left(\alpha / f^{2} \propto \eta_{\mathrm{V}}+\frac{4}{3} \eta_{\mathrm{S}}\right)$. For polymer solutions and melts (Rouse-Zimm dynamics), $\eta_{V}$ is assumed to be proportional to $\eta_{S}\left(\eta_{\mathrm{V}} / \eta_{\mathrm{S}} \simeq\right.$ const. $) .{ }^{55}$ So the discussion for the shear viscosity ${ }^{50}$ can be extended to the mixing rules of the ultrasonic attenuation due to polymer modes (here: the background contribution).

(ii) Fits of eqn. (15) to the data and subtraction of the parameter $B_{T}$ from the measured attenuation. This includes the approximation of a frequency independent background attenuation. The corresponding fits of eqn. (15) to representa-

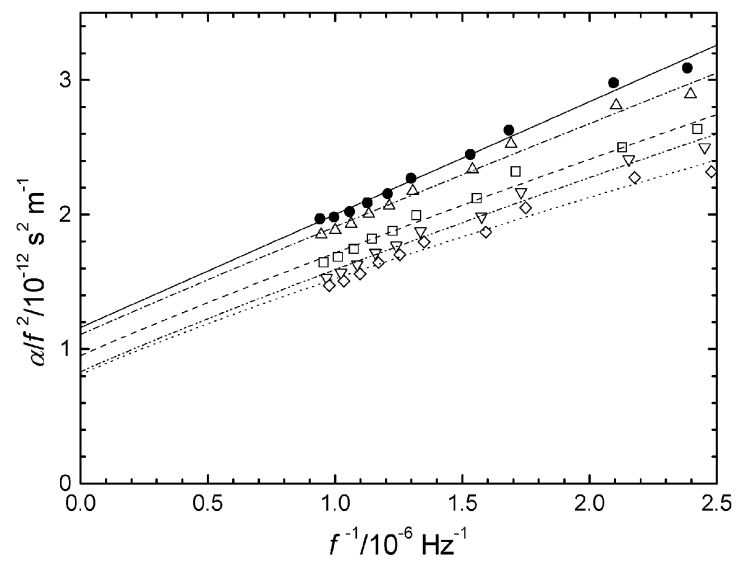

Fig. 7 Ultrasonic attenuation $\alpha / f^{2}$ as a function of $f^{-1}$ at representative temperatures (filled circles: $T=32.8^{\circ} \mathrm{C}$, open up triangles: $T=35^{\circ} \mathrm{C}$, open squares: $T=40^{\circ} \mathrm{C}$, open inverted triangles: $T=45^{\circ} \mathrm{C}$, open diamonds: $T=50^{\circ} \mathrm{C}$ ). The fits of eqn. (15) to the data are represented by dashed and dotted lines. The solid line for $T=32.8^{\circ} \mathrm{C}$ is related to a fit with eqn. (14). The slope $m=$ $8.40 \times 10^{-7} \mathrm{~m} \mathrm{~s}^{-1}$ of the fit (with the measured value $u=$ $1467.0 \mathrm{~m} \mathrm{~s}^{-1}$ ) yields $A=1.23 \times 10^{-3}$. tive data sets are shown in an $\alpha / f^{2} v s . f^{-1}$ plot in Fig. 7. The data are plotted as functions of $f^{-1}$ since the mean field value for the critical exponent of the specific heat is $\tilde{\alpha}=0$ (so, in eqn. (15) $f^{-(1+\tilde{\alpha} /(z \nu))}$ becomes $\left.f^{-1}\right)$. We assume mean field behaviour (in the pseudospinodal concept) for the mixture because of the results from light scattering (see Fig. 3, above).

In Fig. 8, the values for the ultrasonic background attenuation $\left(\alpha / f^{2}\right)_{\text {bg }}$ determined by linear construction (eqn. (23)) and reciprocal construction (eqn. (24)) are both compared with the results for the background attenuation $B_{T}$ by the fits of eqn. (15). The background attenuation $B_{T}$ shows a better accordance with the reciprocal construction than with the arithmetical construction. The same had been shown before for a critical mixture of PEG400/PPG1000. ${ }^{16}$ The reciprocal construction corresponds to the mixing rule for the shear viscosity data of polymer mixtures (see above). The functional form of the mixing rule for the viscosity or the diffusion coefficient with regard to its composition dependence has been in dispute for a long time ${ }^{56}$ known as the "fast" and "slow" mode theories, respectively. The slow mode approach was derived under condition of microscopic incompressibility within the frame of a mean field theory which leads to eqn. (23), ${ }^{57,58}$ whereas the fast mode approach is based on a Hartley-Crank type of equation allowing segment fluxes of different diffusivities leading to eqn. (24). ${ }^{59,60}$ Since the glass temperature $T_{\mathrm{g}}$ is dependent on concentration $\left(T_{\mathrm{g}}=T_{\mathrm{g}}(\phi)\right)$, it is now believed ${ }^{56}$ that the forces ultimately responsible for $T_{\mathrm{g}}$ result in a slow mode type of behaviour which is related to the nearness of $T_{\mathrm{g}}$ to the experimental temperature. So far, a number of experimental studies support this: The slow mode approach is valid for systems close to the glass temperature ${ }^{61}$ and the fast mode approach for systems far away from $T_{\mathrm{g}}{ }^{62}$ The situation here is similar to the latter one, and consequently eqn. (24) is valid here. Therefore we used the reciprocal construction for further analysis of the data.

Fig. 9 shows the measured ultrasonic attenuation $\alpha / f^{2}$ for the highest $\left(T-T_{\mathrm{P}}=17.3 \mathrm{~K}\right)$ and for the lowest $\left(T-T_{\mathrm{P}}=0.1 \mathrm{~K}\right)$ temperature, together with the background attenuation obtained from the contribution of the pure components PEG600 and PPG1000 by using the reciprocal construction (eqn. (24)). As examples, the excess attenuation of the mixture $\left(\alpha / f^{2}\right)_{\text {mix }}$ obtained by this method is shown for three different temperatures in Fig. 10. The data are plotted as functions of $f^{-1}$. As in Fig. 7 (see above), the exponent -1 is justified by the mean field behaviour of the mixture. First we fitted eqn. (14), replacing $\alpha / f^{2}$ by $\left(\alpha / f^{2}\right)_{\text {mix }}$, with $\tilde{\alpha}=0$ and $B=0$, to the data in the vicinity of the phase separation tem-

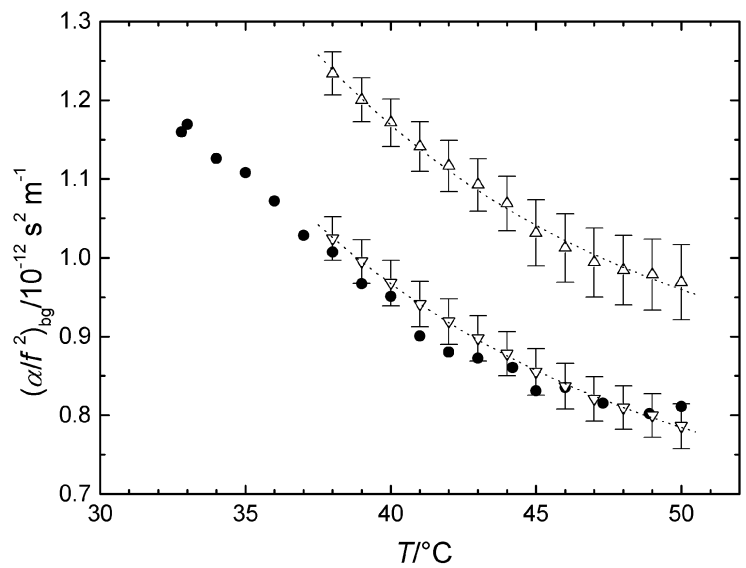

Fig. 8 Temperature dependence of the background attenuation $(\alpha)$ $f^{2}$ ) bg estimated by an arithmetical averaged background (open up triangles) using eqn. (23), by a reciprocal averaged background (open inverted triangles) using eqn. (24), and $\left(\alpha / f^{2}\right)_{\mathrm{bg}}=B_{T}$ estimated by fitting the $\alpha / f^{2}$ data with eqn. (15) (filled circles). 



Fig. 9 Ultrasonic attenuation $\alpha / f^{2}$ as a function of frequency $f$ at $T=50^{\circ} \mathrm{C}$ and $T=32.8^{\circ} \mathrm{C}$. Filled circles: experimental data from the non-critical mixture $\left(y_{\mathrm{PPG}}=0.365\right)$, open down triangles: background attenuation $\left(\alpha / f^{2}\right)_{\text {bg }}$ calculated by a reciprocal averaging (eqn. (24)).

perature $\left(T=32.8^{\circ} \mathrm{C}: T-T_{\mathrm{P}}=0.1 \mathrm{~K}\right)$. This yielded the value of the constant $A$. The approximation $\left(\alpha / f^{2}\right)_{\text {mix }}, T_{\mathrm{p}}+0.1 \mathrm{~K} \simeq(\alpha /$ $\left.f^{2}\right)_{\text {mix, }} T_{\mathrm{PS}}$ is justified because the excess attenuation shows only a weak temperature dependence.

Then we used this value of $A$ for the fits of eqn. (15) to the data for the remaining temperatures, again with $\tilde{\alpha}=0$ and $B_{T}=0$ and replacing $\alpha / f^{2}$ by $\left(\alpha / f^{2}\right)_{\text {mix }}$. From the fits of eqn. (15), we got the frequency of the composition fluctuations $\omega_{D}$ for different temperatures. The temperature dependence of

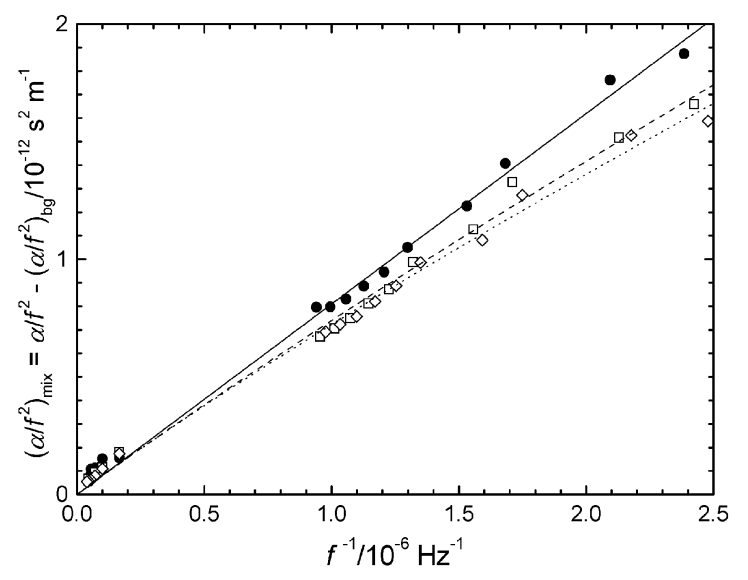

Fig. 10 Excess ultrasonic attenuation of the mixture $\left(\alpha / f^{2}\right)_{\text {mix }}$ at three temperatures as a function of $f^{-1}$ (filled circles: $T=32.8^{\circ} \mathrm{C}$, open squares: $T=40^{\circ} \mathrm{C}$, open diamonds: $T=50^{\circ} \mathrm{C}$ ). The solid line represents a fit of eqn. (14), with $B_{T}=0$, to the data for $T=32.8^{\circ} \mathrm{C}$ (its slope $m=8.10 \times 10^{-7} \mathrm{~m} \mathrm{~s}^{-1}$, with $u=1467.0 \mathrm{~m} \mathrm{~s}^{-1}$, yields $\left.A=1.19 \times 10^{-3}\right)$. The dashed and the dotted lines are relatedto fits of eqn. (15), with $B_{T}=0$, to the data for $T=40^{\circ} \mathrm{C}$ and $T=50^{\circ} \mathrm{C}$, respectively.

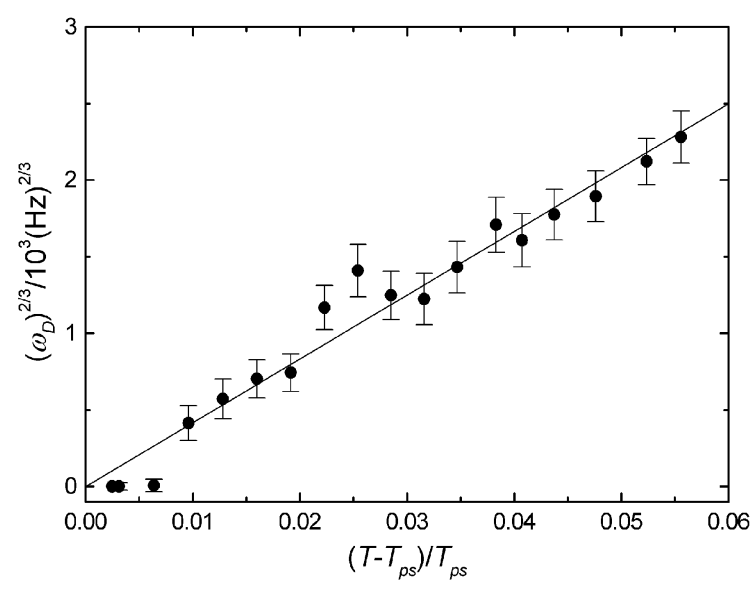

Fig. 11 Characteristic frequency $\left(\omega_{D}\right)^{2 / 3}$ of the composition fluctuations as a function of the reduced temperature $\left(T-T_{\mathrm{PS}}\right) / T_{\mathrm{PS}}$. As a result of a linear fit, $T_{\mathrm{PS}}=32.04{ }^{\circ} \mathrm{C}$ and $\omega_{0}=8.0 \mathrm{MHz}$ are obtained.

the frequency $\omega_{D}$ for a critical mixture is described by the function $\omega_{D}=\omega_{0} \varepsilon^{z \nu}$ with $z \nu=\left(3+x_{\eta}\right) \nu$. For mean field behaviour, the values of the critical exponents are $\nu=0.5$ and $x_{\eta}=0$, leading to a value of $z \nu=\frac{3}{2}$. Here, this description is extended to the non-critical case by using the pseudospinodal concept as described in eqn. (13), with $\omega_{D}^{\text {n.c. }}=\omega_{0}^{\text {n.c. }}$ $\left(\left(T-T_{\mathrm{PS}}\right) / T_{\mathrm{PS}}\right)^{z \nu}$. In this frame, the pseudospinodal temperature $T_{\mathrm{PS}}$ and the amplitude of the relaxation frequency $\omega_{0}$ were determined by a linear fit to a plot of $\left(\omega_{D}\right)^{z \nu}=\left(\omega_{D}\right)^{2 / 3}$ as a function of temperature (shown in Fig. 11). The results were $T_{\mathrm{PS}}=32.04{ }^{\circ} \mathrm{C}$ and $\omega_{0}=8.0 \mathrm{MHz}$ (phase separation temperature determined by ultrasonic measurements: $T_{\mathrm{P}}=32.7^{\circ} \mathrm{C}$ ). The differences between phase separation temperature and pseudospinodal temperature determined by light scattering $\left(T_{\mathrm{P}}-T_{\mathrm{PS}}=0.58 \mathrm{~K}\right)$ and ultrasonic measurements $\left(T_{\mathrm{P}}-\right.$ $\left.T_{\mathrm{PS}}=0.66 \mathrm{~K}\right)$ agree within the experimental error limits.

Fig. 12 shows the analysis of the data by means of the scaling function $G(\Omega)$ of Bhattacharjee and Ferrell (eqn. (13) for the pseudospinodal concept). The ultrasonic attenuation per wavelength $\alpha_{\lambda}$ divided by this quantity at the pseudospinodal temperature $\alpha_{\lambda, T_{\mathrm{PS}}}$ (approximated by $\alpha_{\lambda}, T_{\mathrm{P}}+0.1 \mathrm{~K}$ ) is plotted as a function of the reduced frequency $\Omega_{\text {n.c. }}=\omega /$ $\omega_{D}^{\text {n.c. }}=\omega /\left(\omega_{0}{ }^{\text {n.c. }} \varepsilon^{z \nu}\right)$. For the pseudospinodal temperature in $\varepsilon=\left(T-T_{\mathrm{PS}}\right) / T_{\mathrm{PS}}$, we used the result $T_{\mathrm{PS}}=32.04^{\circ} \mathrm{C}$ from the analysis shown in Fig. 11. A fit of the scaling function $G\left(\Omega_{\text {n.c. }}\right)$ (eqn. (10) with the reduced frequency $\Omega_{\text {n.c. }}$ from eqn.

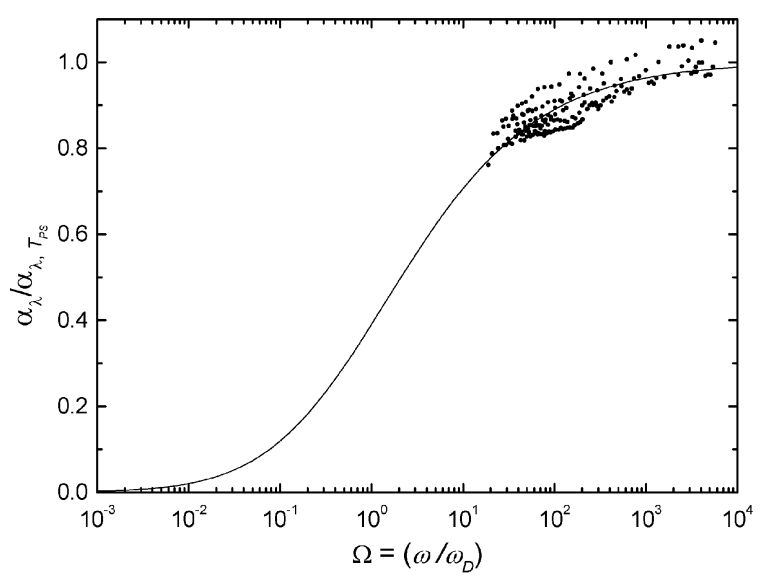

Fig. $12\left(\alpha_{\lambda} / \alpha_{\lambda}, T_{\mathrm{PS}}\right)$ as a function of the reduced frequency $\Omega\left(\alpha_{\lambda}\right.$ : attenuation per wavelength). The solid line was calculated with eqn. (10). 
(13)) to the $\alpha_{\lambda} / \alpha_{\lambda}, T_{\mathrm{PS}}$ data yielded a value of $\omega_{0}=9.4 \mathrm{MHz}$. The small difference to the value of $\omega_{0}=8.0 \mathrm{MHz}$ obtained from the fit in Fig. 11 is due to the slightly different fitting and averaging procedures.

The large difference between the values for $\omega_{0}$ obtained from ultrasonic experiments and the value $\omega_{0}=105 \mathrm{MHz}$ calculated from the light scattering data may arise from the large errors in the ultrasonic experiments and/or the viscosity data used for the calculation of the correlation length from the light scattering data. Furthermore there is a systematic error in the diffusion coefficients due to the differences in the phase diagrams between the samples used for ultrasonic and light scattering experiments. A correction of the light scattering data with reference to this systematic error, using the temperature dependence of the viscosity, reduces the value of $\omega_{0}=105$ $\mathrm{MHz}$ from light scattering to about $40 \mathrm{MHz}$. This difference is, however, still significant and no reasonable explanation exists as yet.

Although the data shown in Fig. 12 are limited to the high frequency part of the scaling function, and in spite of the relative large scatter of the data, there is no fundamental deviation of the data from the scaling function proposed by Bhattacharjee and Ferrell. It should be recognized here again that, in the high frequency limit, the renormalization group calculation of Kroll and Ruhland ${ }^{12}$ gives a similar frequency dependence for the critical mixture.

In the reduced frequency range available in our experiments, the excess attenuation of the non-critical mixture scales (in the pseudospinodal concept) with the same scaling function as the critical mixture ${ }^{16}$ of the same material. It should be noticed that the pseudospinodal concept is rather empirical and that the scaling functions (here: the scaling function of Bhattacharjee and Ferrell) are developed for the vicinity of the critical point. Therefore we can conclude that the distribution of the modes of composition fluctuations which contribute to the ultrasonic attenuation is at least similar for critical and noncritical mixtures.

The combination of the results from a critical mixture ${ }^{16}$ and the non-critical mixture of PEG and PPG yields the following picture:

(i) The distribution of the spectrum of composition fluctuations (distribution of modes) responsible for the ultrasonic excess attenuation of the mixture $\left(\alpha / f^{2}\right)_{\text {mix }}$ seems to be quite general over a large temperature range (critical mixture: $0.1 \leq T-T_{\mathrm{C}} \leq 21.3 \mathrm{~K}$, non-critical mixture: $0.76 \leq T-T_{\mathrm{PS}} \leq$ $17.96 \mathrm{~K}$ ) and also for non-critical compositions. The latter is also justified by an analysis ${ }^{63}$ of ultrasonic data of non-critical mixtures of cyclohexane and aniline ${ }^{5}$ by using both the pseudospinodal concept and the parametric equation of $\operatorname{stat}^{36}$ in a broad composition range of $\left|y_{\text {aniline }}-y_{\text {aniline, }}\right| \leq 0.3$.

(ii) Although there are no obvious differences in the relaxation spectra of critical and non-critical fluctuations, the temperature dependence of the characteristic relaxation frequencies $\omega_{D}$ of the non-critical mixture reflects mean field behaviour, whereas this dependence for the critical mixture is described by Ising exponents, which is in agreement with the dynamic light scattering experiments for both samples.

\section{Conclusion}

To our knowledge, we have shown for the first time that the pseudospinodal concept can be applied to polymer mixtures. Furthermore, we have shown that the scaling function of Bhattacharjee and Ferrell can be successfully extended to the analysis of the ultrasonic attenuation of non-critical polymer mixtures. lows:

In more detail, the main results can be summarized as fol-
(1) The measured excess ultrasonic attenuation of the noncritical mixture of PEG600/PPG1000 can be attributed to the presence of composition fluctuations.

(2) The data from light scattering measurements can be described satisfactorily in the frame of the pseudospinodal concept using mean field critical exponents.

(3) The dynamic scaling theory of Ferrell and Bhattacharjee, developed for the description of the ultrasonic attenuation in the proximity of the critical point, is an appropriate description for the experimental data of a non-critical polymer mixture. This was found before for low molecular weight mixtures and for critical polymer mixtures. Here it is shown that the theory of Ferrell and Bhattacharjee can be formally extended to a non-critical polymer mixture by using the pseudospinodal concept.

(4) The results for the amplitude of the frequency of the concentration fluctuations $\omega_{0}$ from light scattering and ultrasonic measurements are of the same order of magnitude. They are also comparable to the value for the critical mixture PEG400/PPG1000 investigated. ${ }^{16}$ The dependence of the characteristic frequencies $\omega_{D}$ on the reduced temperature $\left(\left(T-T_{\mathrm{C}}\right) / T_{\mathrm{C}}\right.$ or $\left(T-T_{\mathrm{PS}}\right) / T_{\mathrm{PS}}$, respectively) obtained from ultrasonic experiments is different for the critical and the non-critical mixture. The difference can be related to mean field behaviour for the non-critical mixture and Ising behaviour for the critical PEG/PPG mixture, as found by dynamic light scattering.

(5) The background contribution to the ultrasonic attenuation due to molecular processes typical for polymers (such as Rouse dynamics) could be separated from the fluctuation contribution assuming additive behaviour for the contributions of chain or cluster modes and the composition fluctuations. This should be studied in more detail in future.

(6) The background contribution of the ultrasonic attenuation arising from chain and/or internal cluster modes was found to follow rather a reciprocal mixing rule than an arithmetical one, in agreement with the "fast" mode approach discussed for the mixing laws of the viscosity.

\section{Acknowledgements}

The authors wish to thank Dr B. Steinhoff, L. Kühne, and Dr W. Mayer for the measurement of the cloud point curves of the mixtures. We also thank K. Rode and R. Ghahary for characterization of the molecular weight distributions. The financial support of the Deutsche Forschungsgemeinschaft (Grant No. Al 396/1-2) is gratefully acknowledged.

\section{References}

1 K. F. Herzfeld and T. A. Litovitz, Absorption and Dispersion of Ultrasonic Waves, New York, 1959.

2 (a) C. W. Garland and G. Sanchez, J. Chem. Phys., 1983, 79, 3090; (b) G. Sanchez and C. W. Garland, J. Chem. Phys., 1983, 79, 3100 .

3 D. Woermann, Prog. Colloid Polym. Sci., 1991, 84, 165.

4 G. Jaschull, H. Dunker and D. Woermann, Ber. Bunsen-Ges. Phys. Chem., 1984, 88, 630 .

5 E. Bittmann, I. Alig and D. Woermann, Ber. Bunsen-Ges. Phys. Chem., 1994, 98, 189.

6 L. A. Zubkov, Yu. S. Manucharov, S. A. Manucharova and R. K. Turniyasov, Vysokomol. Soedin. A, 1987, 29, 1932.

7 D. B. Fenner, J. Chem. Phys, 1987, 87, 2377.

8 U. Kaatze and U. Schreiber, Chem. Phys. Lett., 1988, 148, 241.

9 K. Kawasaki, Phys. Rev. A, 1970, 1, 1750.

10 (a) Y. Shiwa and K. Kawasaki, Prog. Theor. Phys, 1981, 66, 118 (b) Y. Shiwa and K. Kawasaki, Prog. Theor. Phys., 1981, 66, 406.

11 (a) J. K. Bhattacharjee and R. A. Ferrell, Phys. Rev. A, 1981, 24, 1643; (b) R. A. Ferrell and J. K. Bhattacharjee, Phys. Rev. A, 1985, 31, 1788. 
12 D. M. Kroll and J. M. Ruhland, Phys. Rev. A, 1981, 23, 371.

13 M. Fixman, J. Chem. Phys., 1962, 36, 1961.

14 L. Mistura, J. Chem. Phys., 1972, 57, 2311.

15 H. Tanaka and Y. Wada, Phys. Rev. A, 1985, 32, 512.

16 W. Mayer, S. Hoffmann, G. Meier and I. Alig, Phys. Rev. E, 1997, 55, 3102 .

17 S. Fusenig and D. Woermann, Ber. Bunsen-Ges. Phys. Chem., 1993, 97, 577.

18 J. Schmitz, L. Belkoura and D. Woermann, J. Chem. Phys., 1994, 101, 476.

19 J. Schmitz, Inaugural-Dissertation (PhD), Universität Köln, 1994.

20 J. P. Rakotoniaina, L. Belkoura and D. Woermann, Z. Phys. Chem., 1996, 196, 237.

21 B. Chu, F. J. Schoenes and M. E. Fisher, Phys. Rev., 1969, 185, 219.

22 Y. Harada, Proceeding of the International Symposium on Dynamics of Ordering Processes in Condensed Matter, August 27-30, 1987, ed. S. Komura and H. Furukawa, Plenum Press, New York, London, 1987, p. 537.

23 P. Schofield, Phys. Rev. Lett., 1969, 22, 606.

24 B. D. Josephson, J. Phys. C, 1969, 2, 1113.

25 R. A. Ferrell, Int. J. Thermophys., 1989, 10, 369.

26 C. A. Tracy and B. M. McCoy, Phys. Rev. B, 1975, 12, 368

27 H. Swinney and D. Henry, Phys. Rev. A, 1973, 8, 2586.

28 D. W. Oxtoby and W. M. Gelbart, J. Chem. Phys., 1974, 61, 2957.

29 K. Kawasaki, Ann. Phys., 1970, 61, 1.

30 R. Folk and G. Moser, Phys. Rev. Lett., 1995, 75, 2706

31 T. Ohta, J. Phys. C, 1977, 10, 791.

32 E. G. Siggia, B. I. Halperin and P. C. Hohenberg, Phys. Rev. B, 1976, 13, 2110.

33 G. B. Benedek, in Polarization, Matière et Rayonnement: Vol. jubilaire en l'honneur d'Alfred Kastler, ed. Société Francaise de Physique, Presses universitaires de France, Paris, 1969.

34 K. Hamano, T. Kawazura, T. Koyama and N. Kuwahara, J. Chem. Phys., 1985, 82, 2718.

35 V. A. Rykov, Russ. J. Phys. Chem., 1985, 59, 464.

36 J. T. Ho and J. D. Litster, Phys. Rev. B, 1970, 2, 4523.

37 B. Steinhoff, M. Rüllmann, L. Kühne and I. Alig, J. Chem. Phys, 1997, 107, 5217.
38 A. Z. Akcasu, in Dynamic Light Scattering, ed. W. Brown, Clarendon Press, Oxford, 1993.

39 F. Eggers and Th. Funck, Rev. Sci. Instrum., 1973, 44, 969.

40 R. Koningsveld, L. A. Kleintjens and H. M. Schoffeleers, Pure Appl. Chem., 1974, 39, 1.

41 M. T. Rätzsch and H. Kehlen, Prog. Polym. Sci., 1989, 14, 1.

42 G. Beaucage and R. S. Stein, Macromolecules, 1993, 26, 1603.

43 O. E. Philippova, S. I. Kuchanov, I. N. Topchieva and V. A. Kabanov, Macromolecules, 1985, 18, 1628.

44 V. N. Kuznetsov, L. V. Lesnevskaya, V. A. Petrova, V. B. Kogan and M. S. Vilesova, Polym. Sci. USSR, 1969, 11, 239.

45 B. I. Lirova, Yu. V. Latychev, N. A. Petrov, A. L. Smolyanskii and A. A. Tager, Polym. Sci. USSR, 1973, 15, 163.

46 I. Alig, S. B. Grigor'ev, Ju. S. Manučarov and S. A. Manučarova, Acta Polym., 1986, 37, 698.

47 I. Alig, E. Donth, W. Schenk, S. Höring and Ch. Wohlfarth, Polymer, 1988, 29, 2081.

48 G. Heinrich, I. Alig and E. Donth, Polymer, 1988, 29, 1198.

49 S. Eckert, G. Meier and I. Alig, Phys. Chem. Chem. Phys., submitted.

50 G. Meier, B. Momper and E. W. Fischer, J. Chem. Phys., 1992, 97, 5884 .

51 S. Eckert, G. Meier and I. Alig, in preparation.

52 D. Landau and E. M. Lifshitz, Fluid Mechanics, Pergamon, London, 1959.

53 P. E. Rouse, J. Chem. Phys., 1953, 21, 1273.

54 B. Zimm, J. Chem. Phys., 1956, 24, 269.

55 M. Schulz and I. Alig, J. Chem. Phys., 1992, 97, 2772.

56 A. Z. Akcasu, Macromol. Theory Simul., 1997, 6, 679.

57 P. G. de Gennes, J. Chem. Phys., 1980, 72, 4756.

58 F. Brochard and P. G. de Gennes, Physica A, 1983, 118, 289.

59 E. J. Kramer, P. Green and C. J. Palmstrøm, Polymer, 1984, 25, 473.

60 H. Sillescu, Macromol. Chem. Rapid Commun., 1984, 5, 519; H. Sillescu, Macromol. Chem. Rapid Commun., 1987, 8, 393.

61 M. G. Brereton, E. W. Fischer, G. Fytas and U. Murschall, J. Chem. Phys., 1987, 86, 5174.

62 G. Meier, G. Fytas, B. Momper and G. Fleischer, Macromolecules, 1993, 26, 5310.

63 I. Alig, E. Bittmann and D. Woermann, unpublished data. 